\title{
A parallel algorithm for solving linear parabolic evolution equations
}

\author{
Raymond van Venetië and Jan Westerdiep \\ Korteweg-de Vries (KdV) Institute for Mathematics, University of Amsterdam, \\ PO Box 94248, 1090 GE Amsterdam, The Netherlands \\ r.vanvenetie@uva.nl, j.h.westerdiep@uva.nl
}

\begin{abstract}
We present an algorithm for the solution of a simultaneous space-time discretization of linear parabolic evolution equations with a symmetric differential operator in space. Building on earlier work, we recast this discretization into a Schur-complement equation whose solution is a quasi-optimal approximation to the weak solution of the equation at hand. Choosing a tensor-product discretization, we arrive at a remarkably simple linear system. Using wavelets in time and standard finite elements in space, we solve the resulting system in linear complexity on a single processor, and in polylogarithmic complexity when parallelized in both space and time. We complement these theoretical findings with large-scale parallel computations showing the effectiveness of the method.
\end{abstract}

Keywords: parabolic PDEs; space-time variational formulations; optimal preconditioning; parallel algorithms; massively parallel computing.

Supplementary material: Source code is available at vVW20b.

\section{Introduction}

This paper deals with solving parabolic evolution equations in a time-parallel fashion using tensor-product discretizations. Time-parallel algorithms for solving parabolic evolution equations have become a focal point following the enormous increase in parallel computing power. Spatial parallelism is a ubiquitous component in large-scale computations, but when spatial parallelism is exhausted, parallelization of the time axis is of interest.

Time-stepping methods first discretize the problem in space, and then solve the arising system of coupled ODEs sequentially, immediately revealing a primary source of difficulty for time-parallel computation.

Alternatively, one can solve simultaneously in space and time. Originally introduced in BJ89|BJ90, these space-time methods are very flexible: some can guarantee quasi-best approximations, meaning that their error is proportional to that of the best approximation from the trial space And13 DS18|FK19|SZ20, or drive adaptive routines [SY18]RS19]. Many are especially well-suited for timeparallel computation GN16[NS19. Since the first significant contribution to time-parallel algorithms [Nie64] in 1964, many methods suitable for parallel computation have surfaced; see the review [Gan15]. 
Parallel complexity. The (serial) complexity of an algorithm measures asymptotic runtime on a single processor in terms of the input size. Parallel complexity measures asymptotic runtime given sufficiently many parallel processors having access to a shared memory, i.e., assuming there are no communication costs.

In the current context of tensor-product discretizations of parabolic PDEs, we denote with $N_{t}$ and $N_{\mathbf{x}}$ the number of unknowns in time and space respectively.

The parareal method LMT01 aims at time-parallelism by alternating a serial coarse-grid solve with fine-grid computations in parallel. This way, each iteration has a time-parallel complexity of $\mathcal{O}\left(\sqrt{N_{t}} N_{\mathbf{x}}\right)$, and combined with parallel multigrid in space, a parallel complexity of $\mathcal{O}\left(\sqrt{N_{t}} \log N_{\mathbf{x}}\right)$. The popular MGRIT algorithm extends these ideas to multiple levels in time; cf. [FFK ${ }^{+} 14$.

Recently, Neumüller and Smears proposed an iterative algorithm that uses a Fast Fourier Transform in time. Each iteration runs serially in $\mathcal{O}\left(N_{t} \log \left(N_{t}\right) N_{\mathbf{x}}\right)$ and parallel in time, in $\mathcal{O}\left(\log \left(N_{t}\right) N_{\mathbf{x}}\right)$. By also incorporating parallel multigrid in space, its parallel runtime may be reduced to $\mathcal{O}\left(\log N_{t}+\log N_{\mathbf{x}}\right)$.

Our contribution. In this paper, we study a variational formulation introduced in [SW20] which was based on work by Andreev And13 And16. Recently in $[$ SvVW21|vVW20a, we studied this formulation in the context of space-time adaptivity and its efficient implementation in serial and on shared-memory parallel computers. The current paper instead focuses on its massively parallel implementation and time-parallel performance.

Our method has remarkable similarities with the approach of [NS19], and the most essential difference is the substitution of their Fast Fourier Transform by our Fast Wavelet Transform. The strengths of both methods include a solid inf-sup theory that enables quasi-optimal approximate solutions from the trial space, ease of implementation, and excellent parallel performance in practice.

Our method has another strength: based on a wavelet transform, for fixed algebraic tolerance it runs serially in linear complexity. Parallel in time, it runs in complexity $\mathcal{O}\left(\log \left(N_{t}\right) N_{\mathbf{x}}\right)$; parallel in space and time, in $\mathcal{O}\left(\log \left(N_{t} N_{\mathbf{x}}\right)\right)$. Moreover, when solving to an algebraic error proportional to the discretization error, incorporating a nested iteration (cf. [Hac85, Ch. 5]) results in complexities $\mathcal{O}\left(N_{t} N_{\mathbf{x}}\right), \mathcal{O}\left(\log \left(N_{t}\right) N_{\mathbf{x}}\right)$, and $\mathcal{O}\left(\log ^{2}\left(N_{t} N_{\mathbf{x}}\right)\right)$ respectively. This is on par with best-known results on parallel complexity for elliptic problems; see also [Bra81].

Organization of this paper. In $₫ 2$, we formally introduce the problem, derive a saddle-point formulation, and provide sufficient conditions for quasi-optimality of discrete solutions. In $\$ 3$, we detail on the efficient computation of these discrete solutions. In $\$$ we take a concrete example - the reaction-diffusion equationand analyze the serial and parallel complexity of our algorithm. In \$5 we test these theoretical findings in practice. We conclude in $\$ 6$.

Notations. For normed linear spaces $U$ and $V$, in this paper for convenience over $\mathbb{R}, \mathcal{L}(U, V)$ will denote the space of bounded linear mappings $U \rightarrow V$ 
endowed with the operator norm $\|\cdot\|_{\mathcal{L}(U, V)}$. The subset of invertible operators in $\mathcal{L}(U, V)$ with inverses in $\mathcal{L}(V, U)$ will be denoted as $\mathcal{L}$ is $(U, V)$.

Given a finite-dimensional subspace $U^{\delta}$ of a normed linear space $U$, we denote the trivial embedding $U^{\delta} \rightarrow U$ by $E_{U}^{\delta}$. For a basis $\Phi^{\delta}$ _viewed formally as a column vector - of $U^{\delta}$, we define the synthesis operator as

$$
\mathcal{F}_{\Phi^{\delta}}: \mathbb{R}^{\operatorname{dim} U^{\delta}} \rightarrow U^{\delta}: \mathbf{c} \mapsto \mathbf{c}^{\top} \Phi^{\delta}=: \sum_{\phi \in \Phi^{\delta}} c_{\phi} \phi
$$

Equip $\mathbb{R}^{\operatorname{dim} U^{\delta}}$ with the Euclidean inner product and identify $\left(\mathbb{R}^{\operatorname{dim} U^{\delta}}\right)^{\prime}$ with $\mathbb{R}^{\operatorname{dim} U^{\delta}}$ using the corresponding Riesz map. We find the adjoint of $\mathcal{F}_{\Phi^{\delta}}$, the analysis operator, to satisfy

$$
\left(\mathcal{F}_{\Phi^{\delta}}\right)^{\prime}:\left(U^{\delta}\right)^{\prime} \rightarrow \mathbb{R}^{\operatorname{dim} U^{\delta}}: f \mapsto f\left(\Phi^{\delta}\right):=[f(\phi)]_{\phi \in \Phi^{\delta}} .
$$

For quantities $f$ and $g$, by $f \lesssim g$, we mean that $f \leq C \cdot g$ with a constant that does not depend on parameters that $f$ and $g$ may depend on. By $f \approx g$, we mean that $f \lesssim g$ and $g \lesssim f$. For matrices $\mathbf{A}$ and $\mathbf{B} \in \mathbb{R}^{N \times N}$, by $\mathbf{A} \approx \mathbf{B}$ we will denote spectral equivalence, i.e. $\mathbf{x}^{\top} \mathbf{A} \mathbf{x} \sim \mathbf{x}^{\top} \mathbf{B} \mathbf{x}$ for all $\mathbf{x} \in \mathbb{R}^{N}$.

\section{Quasi-optimal approximations to the parabolic problem}

Let $V, H$ be separable Hilbert spaces of functions on some spatial domain such that $V$ is continuously embedded in $H$, i.e. $V \hookrightarrow H$, with dense compact embedding. Identifying $H$ with its dual yields the Gelfand triple $V \hookrightarrow H \simeq H^{\prime} \hookrightarrow V^{\prime}$.

For a.e.

$$
t \in I:=(0, T)
$$

let $a(t ; \cdot, \cdot)$ denote a bilinear form on $V \times V$ so that for any $\eta, \zeta \in V, t \mapsto a(t ; \eta, \zeta)$ is measurable on $I$, and such that for a.e. $t \in I$,

$$
\begin{aligned}
& |a(t ; \eta, \zeta)| \lesssim\|\eta\|_{V}\|\zeta\|_{V} \quad(\eta, \zeta \in V) \quad \text { (boundedness), } \\
& a(t ; \eta, \eta) \gtrsim\|\eta\|_{V}^{2} \quad(\eta \in V) \quad \text { (coercivity). }
\end{aligned}
$$

With $(A(t) \cdot)(\cdot):=a(t ; \cdot, \cdot) \in \mathcal{L} \operatorname{is}\left(V, V^{\prime}\right)$, given a forcing function $g$ and initial value $u_{0}$, we want to solve the parabolic initial value problem of

$$
\text { finding } u: I \rightarrow V \text { such that }\left\{\begin{aligned}
\frac{\mathrm{d} u}{\mathrm{~d} t}(t)+A(t) u(t) & =g(t) \quad(t \in I), \\
u(0) & =u_{0} .
\end{aligned} \quad\right.
$$

\subsection{An equivalent self-adjoint saddle-point system}

In a simultaneous space-time variational formulation, the parabolic problem reads as finding $u$ from a suitable space of functions of time and space s.t.

$$
(B w)(v):=\int_{I}\left\langle\frac{\mathrm{d} w}{\mathrm{~d} t}(t), v(t)\right\rangle_{H}+a(t ; w(t), v(t)) \mathrm{d} t=\int_{I}\langle g(t), v(t)\rangle_{H}=: g(v)
$$

for all $v$ from another suitable space of functions of time and space. One possibility to enforce the initial condition is by testing against additional test functions. 
Theorem 1 ([SS09]). With $X:=L_{2}(I ; V) \cap H^{1}\left(I ; V^{\prime}\right), Y:=L_{2}(I ; V)$, we have

$$
\left[\begin{array}{l}
B \\
\gamma_{0}
\end{array}\right] \in \mathcal{L} \operatorname{is}\left(X, Y^{\prime} \times H\right)
$$

where for $t \in \bar{I}, \gamma_{t}: u \mapsto u(t, \cdot)$ denotes the trace map. In other words,

$$
\text { finding } u \in X \text { s.t. }\left(B u, \gamma_{0} u\right)=\left(g, u_{0}\right) \quad \text { given } \quad\left(g, u_{0}\right) \in Y^{\prime} \times H
$$

is a well-posed simultaneous space-time variational formulation of (1).

We define $A \in \mathcal{L}$ is $\left(Y, Y^{\prime}\right)$ and $\partial_{t} \in \mathcal{L}$ is $\left(X, Y^{\prime}\right)$ as

$$
(A u)(v):=\int_{I} a(t ; u(t), v(t)) \mathrm{d} t, \quad \text { and } \quad \partial_{t}:=B-A .
$$

Following [SW20, we assume that $A$ is symmetric. We can reformulate 2 as the self-adjoint saddle point problem

$$
\text { finding }(v, \sigma, u) \in Y \times H \times X \text { s.t. } \quad\left[\begin{array}{ccc}
A & 0 & B \\
0 & \operatorname{Id} & \gamma_{0} \\
B^{\prime} & \gamma_{0}^{\prime} & 0
\end{array}\right]\left[\begin{array}{c}
v \\
\sigma \\
u
\end{array}\right]=\left[\begin{array}{c}
g \\
u_{0} \\
0
\end{array}\right] \text {. }
$$

By taking a Schur complement w.r.t. the $H$-block, we can reformulate this as

$$
\text { finding }(v, u) \in Y \times X \text { s.t. } \quad\left[\begin{array}{cc}
A & B \\
B^{\prime} & -\gamma_{0}^{\prime} \gamma_{0}
\end{array}\right]\left[\begin{array}{l}
v \\
u
\end{array}\right]=\left[\begin{array}{c}
g \\
-\gamma_{0}^{\prime} u_{0}
\end{array}\right] \text {. }
$$

We equip $Y$ and $X$ with 'energy'-norms

$$
\|\cdot\|_{Y}^{2}:=(A \cdot)(\cdot), \quad\|\cdot\|_{X}^{2}:=\left\|\partial_{t} \cdot\right\|_{Y^{\prime}}^{2}+\|\cdot\|_{Y}^{2}+\left\|\gamma_{T} \cdot\right\|_{H}^{2},
$$

which are equivalent to the canonical norms on $Y$ and $X$.

\subsection{Uniformly quasi-optimal Galerkin discretizations}

Our numerical approximations will be based on the saddle-point formulation (4). Let $\left(Y^{\delta}, X^{\delta}\right)_{\delta \in \Delta}$ be a collection of closed subspaces of $Y \times X$ satisfying

$$
X^{\delta} \subset Y^{\delta}, \quad \partial_{t} X^{\delta} \subset Y^{\delta} \quad(\delta \in \Delta),
$$

and

$$
1 \geq \gamma_{\Delta}:=\inf _{\delta \in \Delta} \inf _{0 \neq u \in X^{\delta}} \sup _{0 \neq v \in Y^{\delta}} \frac{\left(\partial_{t} u\right)(v)}{\left\|\partial_{t} u\right\|_{Y^{\prime}}\|v\|_{Y}}>0 .
$$

Remark 2. In [SW20, §4], these conditions were verified for $X^{\delta}$ and $Y^{\delta}$ being tensor-products of (locally refined) finite element spaces in time and space. In SvVW21, we relax these conditions to $X_{t}^{\delta}$ and $Y^{\delta}$ being adaptive sparse grids, allowing adaptive refinement locally in space and time simultaneously. 
For $\delta \in \Delta$, let $\left(v^{\delta}, \bar{u}^{\delta}\right) \in Y^{\delta} \times X^{\delta}$ solve the Galerkin discretization of (4):

$$
\left[\begin{array}{cc}
E_{Y}^{{ }^{\prime}}{ }^{\prime} A E_{Y}^{\delta} & E_{Y}^{\delta}{ }^{\prime} B E_{X}^{\delta} \\
E_{X}^{\delta}{ }^{\prime} B^{\prime} E_{Y}^{\delta} & -E_{X}^{\delta}{ }^{\prime} \gamma_{0}^{\prime} \gamma_{0} E_{X}^{\delta}
\end{array}\right]\left[\begin{array}{c}
v^{\delta} \\
\bar{u}^{\delta}
\end{array}\right]=\left[\begin{array}{c}
E_{Y}^{{ }^{\prime}}{ }^{\prime} g \\
-E_{X}^{{ }^{\prime}}{ }^{\prime} \gamma_{0}^{\prime} u_{0}
\end{array}\right] .
$$

The solution $\left(v^{\delta}, \bar{u}^{\delta}\right)$ of 77 exists uniquely, and exhibits uniform quasi-optimality in that $\left\|u-\bar{u}^{\delta}\right\|_{X} \leq \gamma_{\Delta}^{-\mathrm{T}} \inf _{u_{\delta} \in X^{\delta}}\left\|u-u_{\delta}\right\|_{X}$ for all $\delta \in \Delta$.

Instead of solving a matrix representation of (7) using e.g. preconditioned MINRES, we will opt for a computationally more attractive method. By taking the Schur complement w.r.t. the $Y^{\delta}$-block in $(7)$, and replacing $\left(E_{Y}^{\delta^{\prime}} A E_{Y}^{\delta}\right)^{-1}$ in the resulting formulation by a preconditioner $K_{Y}^{\delta}$ that can be applied cheaply, we arrive at the Schur complement formulation of finding $u^{\delta} \in X^{\delta}$ s.t.

$$
\underbrace{E_{X}^{\delta}{ }^{\prime}\left(B^{\prime} E_{Y}^{\delta} K_{Y}^{\delta} E_{Y}^{\delta}{ }^{\prime} B+\gamma_{0}^{\prime} \gamma_{0}\right) E_{X}^{\delta}}_{=: S^{\delta}} u^{\delta}=\underbrace{E_{X}^{\delta}{ }^{\prime}\left(B^{\prime} E_{Y}^{\delta} K_{Y}^{\delta} E_{Y}^{\delta}{ }^{\prime} g+\gamma_{0}^{\prime} u_{0}\right)}_{=: f^{\delta}} \text {. }
$$

The resulting operator $S^{\delta} \in \mathcal{L}$ is $\left(X^{\delta}, X^{\delta^{\prime}}\right)$ is self-adjoint and elliptic. Given a self-adjoint operator $K_{Y}^{\delta} \in \mathcal{L}\left(Y^{\delta^{\prime}}, Y^{\delta}\right)$ satisfying, for some $\kappa_{\Delta} \geq 1$,

$$
\frac{\left(\left(K_{Y}^{\delta}\right)^{-1} v\right)(v)}{(A v)(v)} \in\left[\kappa_{\Delta}^{-1}, \kappa_{\Delta}\right] \quad\left(\delta \in \Delta, \quad v \in Y^{\delta}\right),
$$

the solution $u^{\delta}$ of $(8)$ exists uniquely as well. In fact, the following holds.

Theorem 3 ([SW20, Rem. 3.8]). Take $\left(Y^{\delta} \times X^{\delta}\right)_{\delta \in \Delta}$ satisfying (5)-(6), and $K_{Y}^{\delta}$ satisfying (9). Solutions $u^{\delta} \in X^{\delta}$ of (8) are uniformly quasi-optimal, i.e.

$$
\left\|u-u^{\delta}\right\|_{X} \leq \frac{\kappa_{\Delta}}{\gamma_{\Delta}} \inf _{u_{\delta} \in X^{\delta}}\left\|u-u_{\delta}\right\|_{X} \quad(\delta \in \Delta) .
$$

\section{Solving efficiently on tensor-product discretizations}

From now on, we assume that $X^{\delta}:=X_{t}^{\delta} \otimes X_{\mathbf{x}}^{\delta}$ and $Y^{\delta}:=Y_{t}^{\delta} \otimes Y_{\mathbf{x}}^{\delta}$ are tensorproducts, and for ease of presentation, we assume that the spatial discretizations on $X^{\delta}$ and $Y^{\delta}$ coincide, i.e. $X_{\mathbf{x}}^{\delta}=Y_{\mathbf{x}}^{\delta}$, reducing (5) to $X_{t}^{\delta} \subset Y_{t}^{\delta}$ and $\frac{\mathrm{d}}{\mathrm{d} t} X_{t}^{\delta} \subset Y_{t}^{\delta}$.

We equip $X_{t}^{\delta}$ with a basis $\Phi_{t}^{\delta}, X_{\mathbf{x}}^{\delta}$ with $\Phi_{\mathbf{x}}^{\delta}$, and $Y_{t}^{\delta}$ with $\Xi^{\delta}$.

\subsection{Construction of $K_{Y}^{\delta}$}

Define $\mathbf{O}:=\left\langle\Xi^{\delta}, \Xi^{\delta}\right\rangle_{L_{2}(I)}$ and $\mathbf{A}_{\mathbf{x}}:=\left\langle\Phi_{\mathbf{x}}^{\delta}, \Phi_{\mathbf{x}}^{\delta}\right\rangle_{V}$. Given $\mathbf{K}_{\mathbf{x}} \approx \mathbf{A}_{\mathbf{x}}^{-1}$ uniformly in $\delta \in \Delta$, define

$$
\mathbf{K}_{Y}:=\mathbf{O}^{-1} \otimes \mathbf{K}_{\mathbf{x}}
$$

Then, the preconditioner $K_{Y}^{\delta}:=\mathcal{F}_{\Xi^{\delta} \otimes \Phi_{\mathbf{x}}^{\delta}} \mathbf{K}_{Y}\left(\mathcal{F}_{\Xi^{\delta} \otimes \Phi_{\mathbf{x}}^{\delta}}\right)^{\prime} \in \mathcal{L}\left(Y^{\delta^{\prime}}, Y^{\delta}\right)$ satisfies (91); cf. [SvVW21, §5.6.1].

When $\Xi^{\delta}$ is orthogonal, $\mathbf{O}$ is diagonal and can be inverted exactly. For standard finite element bases $\Phi_{\mathbf{x}}^{\delta}$, suitable $\mathbf{K}_{\mathbf{x}}$ that can be applied efficiently (at cost linear in the discretization size) are provided by symmetric multigrid methods. 


\subsection{Preconditioning the Schur complement formulation}

We will solve a matrix representation of (8) with an iterative solver, thus requiring a preconditioner. Inspired by the constructions of And16 NS19, we build an optimal self-adjoint coercive preconditioner $K_{X}^{\delta} \in \mathcal{L}\left(X^{\delta^{\prime}}, X^{\delta}\right)$ as a waveletin-time block-diagonal matrix with multigrid-in-space blocks.

Let $U$ be a separable Hilbert space of functions over some domain. A given collection $\Psi=\left\{\psi_{\lambda}\right\}_{\lambda \in \vee_{\Psi}}$ is a Riesz basis for $U$ when

$$
\overline{\operatorname{span} \Psi}=U, \quad \text { and } \quad\|\mathbf{c}\|_{\ell_{2}\left(\vee_{\Psi}\right)} \bar{\sim}\left\|\mathbf{c}^{\top} \Psi\right\|_{U} \quad \text { for all } \mathbf{c} \in \ell_{2}\left(\vee_{\Psi}\right) .
$$

Thinking of $\Psi$ being a basis of wavelet-type, for indices $\lambda \in \vee_{\Psi}$, its level is denoted $|\lambda| \in \mathbb{N}_{0}$. We call $\Psi$ uniformly local when for all $\lambda \in \vee_{\Psi}$,

$\operatorname{diam}\left(\operatorname{supp} \psi_{\lambda}\right) \lesssim 2^{-|\lambda|}$ and $\#\left\{\mu \in \vee_{\Psi}:|\mu|=|\lambda|,\left|\operatorname{supp} \psi_{\mu} \cap \operatorname{supp} \psi_{\lambda}\right|>0\right\} \lesssim 1$.

Assume $\Sigma:=\left\{\sigma_{\lambda}: \lambda \in \vee_{\Sigma}\right\}$ is a uniformly local Riesz basis for $L_{2}(I)$ with $\left\{2^{-|\lambda|} \sigma_{\lambda}: \lambda \in \vee_{\Sigma}\right\}$ Riesz for $H^{1}(I)$. Writing $w \in X$ as $\sum_{\lambda \in \vee_{\Sigma}} \sigma_{\lambda} \otimes w_{\lambda}$ for some $w_{\lambda} \in V$, we define the bounded, symmetric, and coercive bilinear form

$$
\left(D_{X} \sum_{\lambda \in \vee_{\Sigma}} \sigma_{\lambda} \otimes w_{\lambda}\right)\left(\sum_{\mu \in \vee_{\Sigma}} \sigma_{\mu} \otimes v_{\mu}\right):=\sum_{\lambda \in \vee_{\Sigma}}\left\langle w_{\lambda}, v_{\lambda}\right\rangle_{V}+4^{|\lambda|}\left\langle w_{\lambda}, v_{\lambda}\right\rangle_{V^{\prime}}
$$

The operator $D_{X}^{\delta}:=E_{X}^{\delta}{ }^{\prime} D_{X} E_{X}^{\delta}$ is in $\mathcal{L}$ is $\left(X^{\delta}, X^{\delta^{\prime}}\right)$. Its norm and that of its inverse are bounded uniformly in $\delta \in \Delta$. When $X^{\delta}=\operatorname{span} \Sigma^{\delta} \otimes \Phi_{\mathrm{x}}^{\delta}$ for some $\Sigma^{\delta}:=\left\{\sigma_{\lambda}: \lambda \in \vee_{\Sigma^{\delta}}\right\} \subset \Sigma$, the matrix representation of $D_{X}^{\delta}$ w.r.t. $\Sigma^{\delta} \otimes \Phi_{\mathbf{x}}^{\delta}$ is

$$
\left(\mathcal{F}_{\Sigma^{\delta} \otimes \Phi^{\delta}}\right)^{\prime} D_{X}^{\delta} \mathcal{F}_{\Sigma^{\delta} \otimes \Phi^{\delta}}=: \mathbf{D}_{X}^{\delta}=\operatorname{blockdiag}\left[\mathbf{A}_{\mathbf{x}}+4^{|\lambda|}\left\langle\Phi_{\mathbf{x}}^{\delta}, \Phi_{\mathbf{x}}^{\delta}\right\rangle_{V^{\prime}}\right]_{\lambda \in \vee_{\Sigma^{\delta}}} .
$$

Theorem 4 ([SvVW21, §5.6.2]). Define $\mathbf{M}_{\mathbf{x}}:=\left\langle\Phi_{\mathbf{x}}^{\delta}, \Phi_{\mathbf{x}}^{\delta}\right\rangle_{H}$. When we have matrices $\mathbf{K}_{j} \approx\left(\mathbf{A}_{\mathbf{x}}+2^{j} \mathbf{M}_{\mathbf{x}}\right)^{-1}$ uniformly in $\delta \in \Delta$ and $j \in \mathbb{N}_{0}$, it follows that

$$
\mathbf{D}_{X}^{-1} \approx \mathbf{K}_{X}:=\operatorname{blockdiag}\left[\mathbf{K}_{|\lambda|} \mathbf{A}_{\mathbf{x}} \mathbf{K}_{|\lambda|}\right]_{\lambda \in \vee_{\Sigma^{\delta}}} .
$$

This yields an optimal preconditioner $K_{X}^{\delta}:=\mathcal{F}_{\Sigma^{\delta} \otimes \Phi^{\delta}} \mathbf{K}_{X}\left(\mathcal{F}_{\Sigma^{\delta} \otimes \Phi^{\delta}}\right)^{\prime} \in \mathcal{L} \mathrm{is}\left(X^{\delta^{\prime}}, X^{\delta}\right)$.

In OR00 it was shown that under a 'full-regularity' assumption, for quasiuniform meshes, a multiplicative multigrid method yields $\mathbf{K}_{j}$ satisfying the conditions of Thm. 4 which can moreover be applied in linear time.

\subsection{Wavelets in time}

The preconditioner $\mathbf{K}_{X}$ requires $X_{t}^{\delta}$ to be equipped with a wavelet basis $\Sigma^{\delta}$, whereas one typically uses a different (single-scale) basis $\Phi_{t}^{\delta}$ on $X_{t}^{\delta}$. To bridge this gap, a basis transformation from $\Sigma^{\delta}$ to $\Phi_{t}^{\delta}$ is required. We define the wavelet transform as $\mathbf{W}_{t}:=\left(\mathcal{F}_{\Phi_{t}^{\delta}}\right)^{-1} \mathcal{F}_{\Sigma^{\delta}} 1_{1}^{1}$

\footnotetext{
${ }^{1}$ In literature, this transform is typically called an inverse wavelet transform.
} 
Define $V_{j}:=\operatorname{span}\left\{\sigma_{\lambda} \in \Sigma:|\lambda| \leq j\right\}$. Equip each $V_{j}$ with a (single-scale) basis $\Phi_{j}$, and assume that $\Phi_{t}^{\delta}:=\Phi_{J}$ for some $J$, so that $X_{t}^{\delta}:=V_{J}$. Since $V_{j+1}=V_{j} \oplus \operatorname{span} \Sigma_{j}$ where $\Sigma_{j}:=\left\{\sigma_{\lambda}:|\lambda|=j\right\}$, there exist matrices $\mathbf{P}_{j}$ and $\mathbf{Q}_{j}$ such that $\Phi_{j}^{\top}=\Phi_{j+1}^{\top} \mathbf{P}_{j}$ and $\Psi_{j}^{\top}=\Phi_{j+1}^{\top} \mathbf{Q}_{j}$, with $\mathbf{M}_{j}:=\left[\mathbf{P}_{j} \mid \mathbf{Q}_{j}\right]$ invertible.

Writing $v \in V_{J}$ in both forms $v=\mathbf{c}_{0}^{\top} \Phi_{0}+\sum_{j=0}^{J-1} \mathbf{d}_{j}^{\top} \Psi_{j}$ and $v=\mathbf{c}_{J}^{\top} \Phi_{J}$, the basis transformation $\mathbf{W}_{t}:=\mathbf{W}_{J}$ mapping wavelet coordinates $\left(\mathbf{c}_{0}^{\top}, \mathbf{d}_{0}^{\top}, \ldots, \mathbf{d}_{J-1}^{\top}\right)$ to single-scale coordinates $\mathbf{c}_{J}$ satisfies

$$
\mathbf{W}_{J}=\mathbf{M}_{J-1}\left[\begin{array}{cc}
\mathbf{W}_{J-1} & \mathbf{0} \\
\mathbf{0} & \mathbf{I d}
\end{array}\right], \text { and } \quad \mathbf{W}_{0}:=\mathbf{I d} .
$$

Uniform locality of $\Sigma$ implies uniform sparsity of the $\mathbf{M}_{j}$, i.e. with $\mathcal{O}(1)$ nonzeros per row and column. Then, assuming a geometrical increase in $\operatorname{dim} V_{j}$ in terms of $j$, which is true in the concrete setting below, matrix-vector products $\mathbf{x} \mapsto \mathbf{W}_{t} \mathbf{x}$ can be performed (serially) in linear complexity; cf. [Ste03].

\subsection{Solving the system}

The matrix representation of $S^{\delta}$ and $f^{\delta}$ from (8) w.r.t. a basis $\Phi_{t}^{\delta} \otimes \Phi_{\mathbf{x}}^{\delta}$ of $X^{\delta}$ is

$$
\mathbf{S}:=\left(\mathcal{F}_{\Phi_{t}^{\delta} \otimes \Phi_{\mathbf{x}}^{\delta}}\right)^{\prime} S^{\delta} \mathcal{F}_{\Phi_{t}^{\delta} \otimes \Phi_{\mathbf{x}}^{\delta}} \quad \text { and } \quad \mathbf{f}:=\left(\mathcal{F}_{\Phi_{t}^{\delta} \otimes \Phi_{\mathbf{x}}^{\delta}}\right)^{\prime} f^{\delta} .
$$

Envisioning an iterative solver, using $\$ 3.2$ we have a preconditioner in terms of the wavelet-in-time basis $\Sigma^{\delta} \otimes \Phi_{\mathbf{x}}^{\delta}$, with which their matrix representation is

$$
\hat{\mathbf{S}}:=\left(\mathcal{F}_{\Sigma^{\delta} \otimes \Phi_{\mathbf{x}}^{\delta}}\right)^{\prime} S^{\delta} \mathcal{F}_{\Sigma^{\delta} \otimes \Phi_{\mathbf{x}}^{\delta}} \quad \text { and } \quad \hat{\mathbf{f}}:=\left(\mathcal{F}_{\Sigma^{\delta} \otimes \Phi_{\mathbf{x}}^{\delta}}\right)^{\prime} f^{\delta} .
$$

These two forms are related: with the wavelet transform $\mathbf{W}:=\mathbf{W}_{t} \otimes \mathbf{I d}_{\mathbf{x}}$, we have $\hat{\mathbf{S}}=\mathbf{W}^{\top} \mathbf{S W}$ and $\hat{\mathbf{f}}=\mathbf{W}^{\top} \mathbf{f}$, and the matrix representation of 8 becomes

$$
\text { finding } \mathbf{w} \text { s.t. } \hat{\mathbf{S}} \mathbf{w}=\hat{\mathbf{f}} \text {. }
$$

We can then recover the solution in single-scale coordinates as $\mathbf{u}=\mathbf{W} \mathbf{w}$.

We use Preconditioned Conjugate Gradients (PCG), with preconditioner $\mathbf{K}_{X}$, to solve 12 . Given an algebraic error tolerance $\epsilon>0$ and current guess $\mathbf{w}_{k}$, we monitor $\mathbf{r}_{k}^{\top} \mathbf{K}_{X} \mathbf{r}_{k} \leq \epsilon^{2}$ where $\mathbf{r}_{k}:=\hat{\mathbf{f}}-\hat{\mathbf{S}} \mathbf{w}_{k}$. This data is available within PCG, and constitutes a stopping criterium: with $u_{k}^{\delta}:=\mathcal{F}_{\Sigma^{\delta} \otimes \Phi_{\mathrm{x}}^{\delta}} \mathbf{w}_{k} \in X^{\delta}$, we see

$$
\mathbf{r}_{k}^{\top} \mathbf{K}_{X} \mathbf{r}_{k}=\left(f^{\delta}-S^{\delta} u_{k}^{\delta}\right)\left(K_{X}^{\delta}\left(f^{\delta}-S^{\delta} u_{k}^{\delta}\right)\right) \approx\left\|u^{\delta}-u_{k}^{\delta}\right\|_{X}^{2}
$$

with $\approx$ following from SvVW21, (4.12)], so that the algebraic error satisfies $\left\|u^{\delta}-u_{k}^{\delta}\right\|_{X} \lesssim \epsilon$.

\section{A concrete setting: the reaction-diffusion equation}

On a bounded Lipschitz domain $\Omega \subset \mathbb{R}^{d}$, take $H:=L_{2}(\Omega), V:=H_{0}^{1}(\Omega)$, and

$$
a(t ; \eta, \zeta):=\int_{\Omega} \mathbf{D} \nabla \eta \cdot \nabla \zeta+c \eta \zeta \mathrm{d} \mathbf{x}
$$


where $\mathbf{D}=\mathbf{D}^{\top} \in \mathbb{R}^{d \times d}$ is positive definite, and $c \geq 02$ We note that $A(t)$ is symmetric and coercive. W.l.o.g. we take $I:=(0,1)$, i.e. $T:=1$.

Fix $p_{t}, p_{\mathbf{x}} \in \mathbb{N}$. With $\left\{\mathcal{T}_{I}\right\}$ the family of quasi-uniform partitions of $I$ into subintervals, and $\left\{\mathcal{T}_{\Omega}\right\}$ that of conforming quasi-uniform triangulations of $\Omega$, we define $\Delta$ as the collection of pairs $\left(\mathcal{T}_{I}, \mathcal{I}_{\Omega}\right)$. We construct our trial- and test spaces as

$$
X^{\delta}:=X_{t}^{\delta} \otimes X_{\mathbf{x}}^{\delta}, \quad Y^{\delta}:=Y_{t}^{\delta} \otimes X_{\mathbf{x}}^{\delta},
$$

where, with $\mathbb{P}_{p}^{-1}(\mathcal{T})$ denoting the space of piecewise degree- $p$ polynomials on $\mathcal{T}$,

$$
X_{t}^{\delta}:=H^{1}(I) \cap \mathbb{P}_{p_{t}}^{-1}\left(\mathcal{T}_{I}\right), \quad X_{\mathbf{x}}^{\delta}:=H_{0}^{1}(\Omega) \cap \mathbb{P}_{p_{\mathbf{x}}}^{-1}\left(\mathcal{T}_{\Omega}\right), \quad Y_{t}^{\delta}:=\mathbb{P}_{p_{t}}^{-1}\left(\mathcal{T}_{I}\right) .
$$

These spaces satisfy condition (5), with coinciding spatial discretizations on $X^{\delta}$ and $Y^{\delta}$. For this choice of $\Delta$, inf-sup condition (6) follows from [SW20, Thm. 4.3].

For $X_{t}^{\delta}$, we choose $\Phi_{t}^{\delta}$ to be the Lagrange basis of degree $p_{t}$ on $\mathcal{T}_{I}$; for $X_{\mathbf{x}}^{\delta}$, we choose $\Phi_{\mathbf{x}}^{\delta}$ to be that of degree $p_{\mathbf{x}}$ on $\mathcal{T}_{\Omega}$. An orthogonal basis $\Xi^{\delta}$ for $Y_{t}^{\delta}$ may be built as piecewise shifted Legendre polynomials of degree $p_{t}$ w.r.t. $\mathcal{T}_{I}$.

For $p_{t}=1$, one finds a suitable wavelet basis $\Sigma$ in Ste98. For $p_{t}>1$, one can either split the system into lowest- and higher-order parts and perform the transform on the lowest-order part only, or construct higher-order wavelets directly; cf. Dij09.

Owing to the tensor-product structure of $X^{\delta}$ and $Y^{\delta}$ and of the operators $A$ and $\partial_{t}$, the matrix representation of our formulation becomes remarkably simple.

Lemma 5. Define $\mathbf{g}:=\left(\mathcal{F}_{\Xi^{\delta} \otimes \Phi_{\mathbf{x}}^{\delta}}\right)^{\prime} g, \mathbf{u}_{0}:=\Phi_{t}^{\delta}(0) \otimes\left\langle u_{0}, \Phi_{\mathbf{x}}^{\delta}\right\rangle_{L_{2}(\Omega)}$, and

$$
\begin{aligned}
\mathbf{T} & :=\left\langle\frac{\mathrm{d}}{\mathrm{d} t} \Phi_{t}^{\delta}, \Xi^{\delta}\right\rangle_{L_{2}(I)}, & \mathbf{N} & :=\left\langle\Phi_{t}^{\delta}, \Xi^{\delta}\right\rangle_{L_{2}(I)}, \\
\boldsymbol{\Gamma}_{0} & :=\Phi_{t}^{\delta}(0)\left[\Phi_{t}^{\delta}(0)\right]^{\top}, & \mathbf{M}_{\mathbf{x}} & :=\left\langle\Phi_{\mathbf{x}}^{\delta}, \Phi_{\mathbf{x}}^{\delta}\right\rangle_{L_{2}(\Omega)}, \\
\mathbf{A}_{\mathbf{x}} & :=\left\langle\mathbf{D} \nabla \Phi_{\mathbf{x}}^{\delta}, \nabla \Phi_{\mathbf{x}}^{\delta}\right\rangle_{L_{2}(\Omega)}+c \mathbf{M}_{\mathbf{x}}, & \mathbf{B} & :=\mathbf{T} \otimes \mathbf{M}_{\mathbf{x}}+\mathbf{N} \otimes \mathbf{A}_{\mathbf{x}}
\end{aligned}
$$

With $\mathbf{K}_{Y}:=\mathbf{O}^{-1} \otimes \mathbf{K}_{\mathbf{x}}$ from 3.1. we can write $\mathbf{S}$ and $\mathbf{f}$ from 3.4 as

$$
\mathbf{S}=\mathbf{B}^{\top} \mathbf{K}_{Y} \mathbf{B}+\boldsymbol{\Gamma}_{0} \otimes \mathbf{M}_{\mathbf{x}}, \quad \mathbf{f}=\mathbf{B}^{\top} \mathbf{K}_{Y} \mathbf{g}+\mathbf{u}_{0} .
$$

Note that $\mathbf{N}$ and $\mathbf{T}$ are non-square, $\boldsymbol{\Gamma}_{0}$ is very sparse, and $\mathbf{T}$ is bidiagonal.

In fact, assumption (5) allows us to write $\mathbf{S}$ in an even simpler form.

Lemma 6. The matrix $\mathbf{S}$ can be written as

$$
\begin{aligned}
\mathbf{S}=\mathbf{A}_{t} \otimes\left(\mathbf{M}_{\mathbf{x}} \mathbf{K}_{\mathbf{x}} \mathbf{M}_{\mathbf{x}}\right) & +\mathbf{M}_{t} \otimes\left(\mathbf{A}_{\mathbf{x}} \mathbf{K}_{\mathbf{x}} \mathbf{A}_{\mathbf{x}}\right)+\mathbf{L}^{\top} \otimes\left(\mathbf{M}_{\mathbf{x}} \mathbf{K}_{\mathbf{x}} \mathbf{A}_{\mathbf{x}}\right) \\
& +\mathbf{L} \otimes\left(\mathbf{A}_{\mathbf{x}} \mathbf{K}_{\mathbf{x}} \mathbf{M}_{\mathbf{x}}\right)+\mathbf{\Gamma}_{0} \otimes \mathbf{M}_{\mathbf{x}}
\end{aligned}
$$

where

$$
\mathbf{L}:=\left\langle\frac{\mathrm{d}}{\mathrm{d} t} \Phi_{t}^{\delta}, \Phi_{t}^{\delta}\right\rangle_{L_{2}(I)}, \quad \mathbf{M}_{t}:=\left\langle\Phi_{t}^{\delta}, \Phi_{t}^{\delta}\right\rangle_{L_{2}(I)}, \quad \mathbf{A}_{t}:=\left\langle\frac{\mathrm{d}}{\mathrm{d} t} \Phi_{t}^{\delta}, \frac{\mathrm{d}}{\mathrm{d} t} \Phi_{t}^{\delta}\right\rangle_{L_{2}(I)} .
$$

This matrix representation does not depend on $Y_{t}^{\delta}$ or $\Xi^{\delta}$ at all.

\footnotetext{
${ }^{2}$ This is easily generalized to variable coefficients, but notation becomes more obtuse.
} 
Proof. The expansion of $\mathbf{B}:=\mathbf{T} \otimes \mathbf{M}_{\mathbf{x}}+\mathbf{N} \otimes \mathbf{A}_{\mathbf{x}}$ in $\mathbf{S}$ yields a sum of five Kronecker products, one of which is

$$
\left(\mathbf{T}^{\top} \otimes \mathbf{M}_{\mathbf{x}}\right) \mathbf{K}_{Y}\left(\mathbf{T} \otimes \mathbf{A}_{\mathbf{x}}\right)=\left(\mathbf{T}^{\top} \mathbf{O}^{-1} \mathbf{N}\right) \otimes\left(\mathbf{M}_{\mathbf{x}} \mathbf{K}_{\mathbf{x}} \mathbf{A}_{\mathbf{x}}\right) .
$$

We will show that $\mathbf{T}^{\top} \mathbf{O}^{-1} \mathbf{N}=\mathbf{L}^{\top}$; similar arguments hold for the other terms. Thanks to $X_{t}^{\delta} \subset Y_{t}^{\delta}$, we can define the trivial embedding $F_{t}^{\delta}: X_{t}^{\delta} \rightarrow Y_{t}^{\delta}$. Defining

$$
\begin{aligned}
T^{\delta}: X_{t}^{\delta} \rightarrow Y_{t}^{\delta^{\prime}}, & \left(T^{\delta} u\right)(v):=\left\langle\frac{\mathrm{d}}{\mathrm{d} t} u, v\right\rangle_{L_{2}(I)}, \\
M^{\delta}: Y_{t}^{\delta} \rightarrow Y_{t}^{\delta^{\prime}}, & \left(M^{\delta} u\right)(v):=\langle u, v\rangle_{L_{2}(I)},
\end{aligned}
$$

we find $\mathbf{O}=\left(\mathcal{F}_{\Xi^{\delta}}\right)^{\prime} M^{\delta} \mathcal{F}_{\Xi^{\delta}}, \mathbf{N}=\left(\mathcal{F}_{\Xi^{\delta}}\right)^{\prime} M^{\delta} \mathcal{F}_{t}^{\delta} \mathcal{F}_{\Phi_{t}^{\delta}}$ and $\mathbf{T}=\left(\mathcal{F}_{\Xi^{\delta}}\right)^{\prime} T^{\delta} \mathcal{F}_{\Phi_{t}^{\delta}}$, so

$$
\mathbf{T}^{\top} \mathbf{O}^{-1} \mathbf{N}=\left(\mathcal{F}_{\Phi_{t}^{\delta}}\right)^{\prime} T^{\delta^{\prime}} F_{t}^{\delta} \mathcal{F}_{\Phi_{t}^{\delta}}=\left\langle\Phi_{t}, \frac{\mathrm{d}}{\mathrm{d} t} \Phi_{t}\right\rangle_{L_{2}(I)}=\mathbf{L}^{\top}
$$

\subsection{Parallel complexity}

The parallel complexity of our algorithm is the asymptotic runtime of solving (12) for $\mathbf{u} \in \mathbb{R}^{N_{t} N_{\mathbf{x}}}$ in terms of $N_{t}:=\operatorname{dim} X_{t}^{\delta}$ and $N_{\mathbf{x}}:=\operatorname{dim} X_{\mathbf{x}}^{\delta}$, given sufficiently many parallel processors and assuming no communication cost.

We understand the serial (resp. parallel) cost of a matrix $\mathbf{B}$, denoted $C_{\mathbf{B}}^{s}$ (resp. $C_{\mathbf{B}}^{p}$ ), as the asymptotic runtime of performing $\mathbf{x} \mapsto \mathbf{B x} \in \mathbb{R}^{N}$ in terms of $N$, on a single (resp. sufficiently many) processors at no communication cost. For uniformly sparse matrices, i.e. with $\mathcal{O}(1)$ nonzeros per row and column, the serial cost is $\mathcal{O}(N)$, and the parallel cost is $\mathcal{O}(1)$ by computing each cell of the output concurrently.

From Theorem 4, we see that $\mathbf{K}_{X}$ is such that $\kappa_{2}\left(\mathbf{K}_{X} \hat{\mathbf{S}}\right) \lesssim 1$ uniformly in $\delta \in \Delta$. Therefore, for a given algebraic error tolerance $\epsilon$, we require $\mathcal{O}\left(\log \epsilon^{-1}\right)$ PCG iterations. Assuming that the parallel cost of matrices dominates that of vector addition and inner products, the parallel complexity of a single PCG iteration is dominated by the cost of applying $\mathbf{K}_{X}$ and $\hat{\mathbf{S}}$. As $\hat{\mathbf{S}}=\mathbf{W}^{\top} \mathbf{S W}$, our algorithm runs in complexity

$$
\mathcal{O}\left(\log \epsilon^{-1}\left[C_{\mathbf{K}_{X}}^{\circ}+C_{\mathbf{W}^{\top}}^{\circ}+C_{\mathbf{S}}^{\circ}+C_{\mathbf{W}}^{\circ}\right]\right) \quad(\circ \in\{s, p\}) .
$$

Theorem 7. For fixed algebraic error tolerance $\epsilon>0$, our algorithm runs in

- serial complexity $\mathcal{O}\left(N_{t} N_{\mathbf{x}}\right)$;

- time-parallel complexity $\mathcal{O}\left(\log \left(N_{t}\right) N_{\mathbf{x}}\right)$;

- space-time-parallel complexity $O\left(\log \left(N_{t} N_{\mathbf{x}}\right)\right)$.

Proof. We absorb the constant factor $\log \epsilon^{-1}$ of $(14)$ into $\mathcal{O}$. We analyse the cost of every matrix separately. 
The (inverse) wavelet transform. As $\mathbf{W}=\mathbf{W}_{t} \otimes \mathbf{I d}_{\mathbf{x}}$, its serial cost equals $\mathcal{O}\left(C_{\mathbf{W}_{t}}^{s} N_{\mathbf{x}}\right)$. The choice of wavelet allows performing $\mathbf{x} \mapsto \mathbf{W}_{t} \mathbf{x}$ at linear serial cost (cf. 3.3 , so that $C_{\mathrm{W}}^{s}=\mathcal{O}\left(N_{t} N_{\mathbf{x}}\right)$.

Using (10), we write $\mathbf{W}_{t}$ as the composition of $J$ matrices, each uniformly sparse and hence at parallel cost $\mathcal{O}(1)$. Because the mesh in time is quasi-uniform, we have $J \approx \log N_{t}$. We find that $C_{\mathbf{W}_{t}}^{p}=\mathcal{O}(J)=\mathcal{O}\left(\log N_{t}\right)$, so that the timeparallel cost of $\mathbf{W}$ equals $\mathcal{O}\left(\log \left(N_{t}\right) N_{\mathbf{x}}\right)$. By exploiting spatial parallelism as well, we find $C_{\mathbf{W}}^{p}=\mathcal{O}\left(\log N_{t}\right)$. Analogous arguments hold for $\mathbf{W}_{t}^{\top}$ and $\mathbf{W}^{\top}$.

The preconditioner. Recall that $\mathbf{K}_{X}:=\operatorname{blockdiag}\left[\mathbf{K}_{|\lambda|} \mathbf{A}_{\mathbf{x}} \mathbf{K}_{|\lambda|}\right]_{\lambda}$. Since the cost of $\mathbf{K}_{j}$ is independent of $j$, we see that

$$
C_{\mathbf{K}_{X}}^{s}=\mathcal{O}\left(N_{t} \cdot\left(2 C_{\mathbf{K}_{j}}^{s}+C_{\mathbf{A}_{\mathbf{x}}}^{s}\right)\right)=\mathcal{O}\left(2 N_{t} C_{\mathbf{K}_{j}}^{s}+N_{t} N_{\mathbf{x}}\right) .
$$

Implementing the $\mathbf{K}_{j}$ as typical multiplicative multigrid solvers with linear serial cost, we find $C_{\mathbf{K}_{X}}^{s}=\mathcal{O}\left(N_{t} N_{\mathbf{x}}\right)$.

Through temporal parallelism, we can apply each block of $\mathbf{K}_{X}$ concurrently, resulting in a time-parallel cost of $\mathcal{O}\left(2 C_{\mathbf{K}_{j}}^{s}+C_{\mathbf{A}_{\mathbf{x}}}^{s}\right)=\mathcal{O}\left(N_{\mathbf{x}}\right)$.

By parallelizing in space as well, we reduce the cost of the uniformly sparse $\mathbf{A}_{\mathbf{x}}$ to $\mathcal{O}(1)$. The parallel cost of multiplicative multigrid on quasi-uniform triangulations is $\mathcal{O}\left(\log N_{\mathbf{x}}\right)$; cf. $\mathrm{MFL}^{+} 91$. It follows that $C_{\mathbf{K}_{X}}^{p}=\mathcal{O}\left(\log N_{\mathbf{x}}\right)$.

The Schur matrix. Using Lemma 5 , we write $\mathbf{S}=\mathbf{B}^{\top} \mathbf{K}_{Y} \mathbf{B}+\boldsymbol{\Gamma}_{0} \otimes \mathbf{M}_{\mathbf{x}}$ where $\mathbf{B}=\mathbf{T} \otimes \mathbf{M}_{\mathbf{x}}+\mathbf{N} \otimes \mathbf{A}_{\mathbf{x}}$, which immediately reveals that

$$
\begin{aligned}
& C_{\mathbf{S}}^{s}=C_{\mathbf{B}^{\top}}^{s}+C_{\mathbf{K}_{Y}}^{s}+C_{\mathbf{B}}^{s}+C_{\mathbf{\Gamma}_{0}}^{s} \cdot C_{\mathbf{M}}^{s}=\mathcal{O}\left(N_{t} N_{\mathbf{x}}+C_{\mathbf{K}_{Y}}^{s}\right), \quad \text { and } \\
& C_{\mathbf{S}}^{p}=\max \left\{C_{\mathbf{B}^{\top}}^{p}+C_{\mathbf{K}_{Y}}^{p}+C_{\mathbf{B}}^{p}, \quad C_{\mathbf{\Gamma}_{0}}^{p} \cdot C_{\mathbf{M}}^{p}\right\}=\mathcal{O}\left(C_{\mathbf{K}_{Y}}^{p}\right)
\end{aligned}
$$

because every matrix except $\mathbf{K}_{Y}$ is uniformly sparse. With arguments similar to the previous paragraph, we see that $\mathbf{K}_{Y}$ (and hence $\mathbf{S}$ ) has serial $\operatorname{cost} \mathcal{O}\left(N_{t} N_{\mathbf{x}}\right)$, time-parallel cost $\mathcal{O}\left(N_{\mathbf{x}}\right)$, and space-time-parallel cost $\mathcal{O}\left(\log N_{\mathbf{x}}\right)$.

\subsection{Solving to higher accuracy}

Instead of fixing the algebraic error tolerance, maybe more realistic is is to desire a solution $\tilde{u}^{\delta} \in X^{\delta}$ for which the error is proportional to the discretization error, i.e. $\left\|u-\tilde{u}^{\delta}\right\|_{X} \lesssim \inf _{u_{\delta} \in X^{\delta}}\left\|u-u_{\delta}\right\|_{X}$.

Assuming that this error decays with a (problem-dependent) rate $s>0$, i.e. $\inf _{u_{\delta} \in X^{\delta}}\left\|u-u_{\delta}\right\|_{X} \lesssim\left(N_{t} N_{\mathbf{x}}\right)^{-s}$, then the same holds for the solution $u^{\delta}$ of (8); cf. Thm. 3. When the algebraic error tolerance decays as $\epsilon \lesssim\left(N_{t} N_{\mathbf{x}}\right)^{-s}$, a triangle inequality and (13) show that the error of our solution $\tilde{u}^{\delta}$ obtained by PCG decays at rate $s$ too.

In this case, $\log \epsilon^{-1}=\mathcal{O}\left(\log \left(N_{t} N_{\mathbf{x}}\right)\right)$. From (14) and the proof of Theorem 7, we find our algorithm to run in superlinear serial complexity $\mathcal{O}\left(N_{t} N_{\mathbf{x}} \log \left(N_{t} N_{\mathbf{x}}\right)\right)$, time-parallel complexity $\mathcal{O}\left(\log ^{2}\left(N_{t}\right) \log \left(N_{\mathbf{x}}\right) N_{\mathbf{x}}\right)$, and polylogarithmic complexity $\mathcal{O}\left(\log ^{2}\left(N_{t} N_{\mathbf{x}}\right)\right)$ parallel in space and time. 
For elliptic PDEs, algorithms are available that offer quasi-optimal solutions, serially in linear complexity $\mathcal{O}\left(N_{\mathbf{x}}\right)$ - the cost of a serial solve to fixed algebraic error - and in parallel in $\mathcal{O}\left(\log ^{2} N_{\mathbf{x}}\right)$, by combining a nested iteration with parallel multigrid; cf. [Hac85, Ch. 5] and [Bra81].

In [HVW95, the question is posed whether "good serial algorithms for parabolic PDEs are intrinsically as parallel as good serial algorithms for elliptic PDEs", basically asking if the lower bound of $\mathcal{O}\left(\log ^{2}\left(N_{t} N_{\mathbf{x}}\right)\right)$ can be attained by an algorithm that runs serially in $\mathcal{O}\left(N_{t} N_{\mathbf{x}}\right)$; see [Wor91, §2.2] for a formal discussion.

Nested iteration drives down the serial complexity of our algorithm to a linear $\mathcal{O}\left(N_{t} N_{\mathbf{x}}\right)$, and also improves the time-parallel complexity to $\mathcal{O}\left(\log \left(N_{t}\right) N_{\mathbf{x}}\right) !^{3}$ This is on par with the best-known results for elliptic problems, so we answer the question posed in [HVW95] in the affirmative.

\section{$5 \quad$ Numerical experiments}

We take the simple heat equation, i.e. $D=\mathbf{I d}_{\mathbf{x}}$ and $c=0$. We select $p_{t}=p_{\mathbf{x}}=1$, i.e. lowest order finite elements in space and time. We will use the 3-point wavelet introduced in Ste98.

We implemented our algorithm in Python using the open source finite element library NGSolve [Sch14 for meshing and discretization of the bilinear forms in space and time, MPI through mpi4py [DPS05] for distributed computations, and SciPy [Vir20] for the sparse matrix-vector computations. The source code is available at vVW20b.

\subsection{Preconditioner calibration on a 2D problem}

Our wavelet-in-time, multigrid-in-space preconditioner is optimal: $\kappa_{2}\left(\mathbf{K}_{X} \hat{\mathbf{S}}\right) \lesssim 1$. Here we will investigate this condition number quantitatively.

As a model problem, we partition the temporal interval $I$ uniformly into $2^{J}$ subintervals. We consider the domain $\Omega:=[0,1]^{2}$, and triangulate it uniformly into $4^{K}$ triangles. We set $N_{t}:=\operatorname{dim} X_{t}^{\delta}=2^{J}+1$ and $N_{\mathbf{x}}:=\operatorname{dim} X_{\mathbf{x}}^{\delta}=\left(2^{K}-1\right)^{2}$.

We start by using direct inverses $\mathbf{K}_{j}=\left(\mathbf{A}_{\mathbf{x}}+2^{j} \mathbf{M}_{\mathbf{x}}\right)^{-1}$ and $\mathbf{K}_{\mathbf{x}}=\mathbf{A}_{\mathbf{x}}^{-1}$ to determine the best possible condition numbers. We found that replacing $\mathbf{K}_{j}$ by $\mathbf{K}_{j}^{\alpha}=\left(\alpha \mathbf{A}_{\mathbf{x}}+2^{j} \mathbf{M}_{\mathbf{x}}\right)^{-1}$ for $\alpha=0.3$ gave better conditioning; see also the left of Table 5.1. At the right of Table 5.1, we see that the condition numbers are very robust with respect to spatial refinements, but less so for refinements in time. Still, at $N_{t}=16129$, we observe a modest $\kappa_{2}\left(\mathbf{K}_{X} \hat{\mathbf{S}}\right)$ of 8.74 .

Replacing the direct inverses with multigrid solvers, we found a good balance between speed and conditioning at $2 \mathrm{~V}$-cycles with 3 Gauss-Seidel smoothing steps per grid. We decided to use these for our experiments.

\footnotetext{
${ }^{3}$ Interestingly, nested iteration offers no improvements parallel in space and time, with complexity still $\mathcal{O}\left(\log ^{2}\left(N_{t} N_{\mathbf{x}}\right)\right)$.
} 


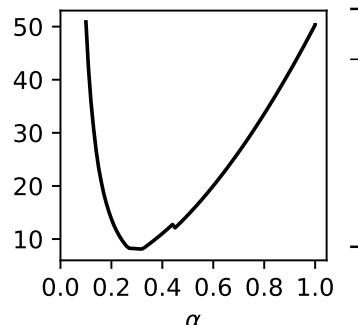

\begin{tabular}{rrrrrrrrr}
\hline \multicolumn{2}{r}{$N_{t}=65$} & 129 & 257 & 513 & 1025 & 2049 & 4097 & 8193 \\
\hline$N_{\mathbf{x}}=49$ & 6.34 & 7.05 & 7.53 & 7.89 & 8.15 & 8.37 & 8.60 & 8.78 \\
225 & 6.33 & 6.89 & 7.55 & 7.91 & 8.14 & 8.38 & 8.57 & 8.73 \\
961 & 6.14 & 6.89 & 7.55 & 7.93 & $\mathbf{8 . 1 5}$ & 8.38 & 8.57 & 8.74 \\
3969 & 6.14 & 7.07 & 7.56 & 7.87 & 8.16 & 8.38 & 8.57 & 8.74 \\
16129 & 6.14 & 6.52 & 7.55 & 7.86 & 8.16 & 8.37 & 8.57 & 8.74 \\
\hline
\end{tabular}

Table 5.1. Computed condition numbers $\kappa_{2}\left(\mathbf{K}_{X} \hat{\mathbf{S}}\right)$. Left: fixed $N_{t}=1025, N_{\mathbf{x}}=961$ for varying $\alpha$. Right: fixed $\alpha=0.3$ for varying $N_{t}$ and $N_{\mathbf{x}}$.

\subsection{Time-parallel results}

We perform computations on Cartesius, the Dutch supercomputer. Each Cartesius node has $64 \mathrm{~GB}$ of memory and 12 cores (at 2 threads per core) running at 2.6GHz. Using the preconditioner detailed above, we iterate PCG on 12 with $\mathbf{S}$ computed as in Lemma 6, until achieving an algebraic error of $\epsilon=10^{-6}$; see also 3.4 For the spatial multigrid solvers, we use 2 V-cycles with 3 Gauss-Seidel smoothing steps per grid.

Memory-efficient time-parallel implementation. For $\mathbf{X} \in \mathbb{R}^{N_{\mathbf{x}} \times N_{t}}$, we define $\operatorname{Vec}(\mathbf{X}) \in \mathbb{R}^{N_{t} N_{\mathbf{x}}}$ as the vector obtained by stacking columns of $\mathbf{X}$ vertically. For memory efficency, we do not build matrices of the form $\mathbf{B}_{t} \otimes \mathbf{B}_{\mathbf{x}}$ appearing in Lemma 6 directly, but instead perform matrix-vector products using the identity

$$
\left(\mathbf{B}_{t} \otimes \mathbf{B}_{\mathbf{x}}\right) \operatorname{Vec}(\mathbf{X})=\operatorname{Vec}\left(\mathbf{B}_{\mathbf{x}}\left(\mathbf{B}_{t} \mathbf{X}^{\top}\right)^{\top}\right)=\left(\mathbf{I d}_{t} \otimes \mathbf{B}_{\mathbf{x}}\right) \operatorname{Vec}\left(\mathbf{B}_{t} \mathbf{X}^{\top}\right) .
$$

Each parallel processor stores only a subset of the temporal degrees of freedom, e.g. a subset of columns of $\mathbf{X}$. When $\mathbf{B}_{t}$ is uniformly sparse, which holds true for all of our temporal matrices, using 15 we can evaluate $\left(\mathbf{B}_{t} \otimes \mathbf{B}_{\mathbf{x}}\right) \operatorname{Vec}(\mathbf{X})$ in $\mathcal{O}\left(C_{\mathbf{B}_{\mathbf{x}}}^{s}\right)$ operations parallel in time: on each parallel processor, we compute 'our' columns of $\mathbf{Y}:=\mathbf{B}_{t} \mathbf{X}^{\top}$ by receiving the necessary columns of $\mathbf{X}$ from neighbouring processors, and then compute $\mathbf{B}_{\mathbf{x}} \mathbf{Y}^{\top}$ without communication.

The preconditioner $\mathbf{K}_{X}$ is block-diagonal, making its time-parallel application trivial. Representing the wavelet transform of 3.3 as the composition of $J$ Kronecker products allows a time-parallel implementation using the above.

2D problem. We select $\Omega:=[0,1]^{2}$ with a uniform triangulation $\mathcal{T}_{\Omega}$, and we triangulate $I$ uniformly into $\mathcal{T}_{I}$. We select the smooth solution $u(t, x, y):=$ $\exp \left(-2 \pi^{2} t\right) \sin (\pi x) \sin (\pi y)$, so the problem has vanishing forcing data $g$.

Table 5.2 details the strong scaling results, i.e. fixing the problem size and increasing the number of processors $P$. We triangulate $I$ into $2^{14}$ time slabs, yielding $N_{t}=16385$ temporal degrees of freedom, and $\Omega$ into $4^{8}$ triangles, yielding a $X_{\mathrm{x}}^{\delta}$ of dimension $N_{\mathrm{x}}=65025$. The resulting system contains 1065434625 degrees of freedom and our solver reaches the algebraic error tolerance after 16 iterations. In perfect strong scaling, the total number of CPU-hours remains constant. Even at 2048 processors, we observe a parallel efficiency of around $92.9 \%$, 
solving this system in a modest 11.7 CPU-hours. Acquiring strong scaling results on a single node was not possible due to memory limitations.

Table 5.3 details the weak scaling results, i.e. fixing the problem size per processor and increasing the number of processors. In perfect weak scaling, the time per iteration should remain constant. We observe a slight increase in time per iteration on a single node, but when scaling to multiple nodes, we observe a near-perfect parallel efficiency of around $96.7 \%$, solving the final system with 4278467585 degrees of freedom in a mere 109 seconds.

3D problem. We select $\Omega:=[0,1]^{3}$, and prescribe the solution $u(t, x, y, z):=$ $\exp \left(-3 \pi^{2} t\right) \sin (\pi x) \sin (\pi y) \sin (\pi z)$, so the problem has vanishing forcing data $g$.

Table 5.4 shows the strong scaling results. We triangulate $I$ uniformly into $2^{14}$ time slabs, and $\Omega$ uniformly into $8^{6}$ tetrahedra. The arising system has $N=4097020095$ unknowns, which we solve to tolerance in 18 iterations. The results are comparable to those in two dimensions, albeit a factor two slower at similar problem sizes.

Table 5.5 shows the weak scaling results for the 3D problem. As in the twodimensional case, we observe excellent scaling properties, and see that the time per iteration is nearly constant.

\section{Conclusion}

We have presented a framework for solving linear parabolic evolution equations massively in parallel. Based on earlier ideas And16 [NS19]SW20, we found a remarkably simple symmetric Schur-complement equation. With a tensor-product discretization of the space-time cylinder using standard finite elements in time and space together with a wavelet-in-time multigrid-in-space preconditioner, we were able to solve the arising systems to fixed accuracy in a uniformly bounded number of PCG steps.

We found that our algorithm runs in linear complexity on a single processor. Moreover, when sufficiently many parallel processors are available and communication is free, its runtime scales logarithmically in the discretization size. These complexity results translate to a highly efficient algorithm in practice.

The numerical experiments serve as a showcase for the described space-time method, and exhibit its excellent time-parallelism by solving a linear system with over 4 billion unknowns in just 109 seconds, using just over 2 thousand parallel processors. By incorporating spatial parallelism as well, we expect these results to scale well to much larger problems.

Although performed in the rather restrictive setting of the heat equation discretized using piecewise linear polynomials on uniform triangulations, the parallel framework already allows solving more general linear parabolic PDEs using polynomials of varying degree on locally refined (tensor-product) meshes. In this more general setting, we envision load balancing to become the main hurdle in achieving good scaling results. 


\begin{tabular}{|c|c|c|c|c|c|c|c|}
\hline$P$ & $N_{t}$ & $N_{\mathrm{x}}$ & $N=N_{t} N_{\mathbf{x}}$ & its & time $(\mathrm{s})$ & time/it (s) & CPU-hrs \\
\hline $1-16$ & 16385 & 65025 & 1065434625 & & Ou & memory & \\
\hline 32 & 16385 & 65025 & 1065434625 & 16 & 1224.85 & 76.55 & 10.89 \\
\hline 64 & 16385 & 65025 & 1065434625 & 16 & 615.73 & 38.48 & 10.95 \\
\hline 128 & 16385 & 65025 & 1065434625 & 16 & 309.81 & 19.36 & 11.02 \\
\hline 256 & 16385 & 65025 & 1065434625 & 16 & 163.20 & 10.20 & 11.61 \\
\hline 512 & 16385 & 65025 & 1065434625 & 16 & 96.54 & 6.03 & 13.73 \\
\hline 512 & 16385 & 65025 & 1065434625 & 16 & 96.50 & 6.03 & 13.72 \\
\hline 1024 & 16385 & 65025 & 1065434625 & 16 & 45.27 & 2.83 & 12.88 \\
\hline 2048 & 16385 & 65025 & 1065434625 & 16 & 20.59 & 1.29 & 11.72 \\
\hline
\end{tabular}

Table 5.2. Strong scaling results for the $2 \mathrm{D}$ problem.

\begin{tabular}{|c|c|c|c|c|c|c|c|c|}
\hline & $P$ & $N_{t}$ & $N_{\mathrm{x}}$ & $N=N_{t} N_{\mathbf{x}}$ & its & time $(\mathrm{s})$ & time/it (s) & CPU-hrs \\
\hline \multirow{5}{*}{ 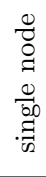 } & 1 & 9 & 261121 & 2350089 & 8 & 33.36 & 4.17 & 0.01 \\
\hline & 2 & 17 & 261121 & 4439057 & 11 & 46.66 & 4.24 & 0.03 \\
\hline & 4 & 33 & 261121 & 8616993 & 12 & 54.60 & 4.55 & 0.06 \\
\hline & 8 & 65 & 261121 & 16972865 & 13 & 65.52 & 5.04 & 0.15 \\
\hline & 16 & 129 & 261121 & 33684609 & 13 & 86.94 & 6.69 & 0.39 \\
\hline \multirow{7}{*}{ 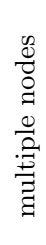 } & 32 & 257 & 261121 & 67108097 & 14 & 93.56 & 6.68 & 0.83 \\
\hline & 64 & 513 & 261121 & 133955073 & 14 & 94.45 & 6.75 & 1.68 \\
\hline & 128 & 1025 & 261121 & 267649025 & 14 & 93.85 & 6.70 & 3.34 \\
\hline & 256 & 2049 & 261121 & 535036929 & 15 & 101.81 & 6.79 & 7.24 \\
\hline & 512 & 4097 & 261121 & 1069812737 & 15 & 101.71 & 6.78 & 14.47 \\
\hline & 1024 & 8193 & 261121 & 2139364353 & 16 & 108.32 & 6.77 & 30.81 \\
\hline & 2048 & 16385 & 261121 & 4278467585 & 16 & 109.59 & 6.85 & 62.34 \\
\hline
\end{tabular}

Table 5.3. Weak scaling results for the $2 \mathrm{D}$ problem.

\begin{tabular}{rrrrrrrr}
\hline$P$ & $N_{t}$ & $N_{\mathbf{x}}$ & $N=N_{t} N_{\mathbf{x}}$ & its & time (s) & time/it (s) & CPU-hrs \\
\hline $1-64$ & 16385 & 250047 & 4097020095 & & out of memory & \\
128 & 16385 & 250047 & 4097020095 & 18 & 3308.49 & 174.13 & 117.64 \\
256 & 16385 & 250047 & 4097020095 & 18 & 1655.92 & 87.15 & 117.75 \\
512 & 16385 & 250047 & 4097020095 & 18 & 895.01 & 47.11 & 127.29 \\
1024 & 16385 & 250047 & 4097020095 & 18 & 451.59 & 23.77 & 128.45 \\
2048 & 16385 & 250047 & 4097020095 & 18 & 221.12 & 12.28 & 125.80 \\
\hline
\end{tabular}

Table 5.4. Strong scaling results for the $3 \mathrm{D}$ problem.

\begin{tabular}{rrrrrrrr}
\hline$P$ & $N_{t}$ & $N_{\mathbf{x}}$ & $N=N_{t} N_{\mathbf{x}}$ & its & time (s) & time/it (s) & CPU-hrs \\
\hline 16 & 129 & 250047 & 32256063 & 15 & 183.65 & 12.24 & 0.82 \\
32 & 257 & 250047 & 64262079 & 16 & 196.26 & 12.27 & 1.74 \\
64 & 513 & 250047 & 128274111 & 16 & 197.55 & 12.35 & 3.51 \\
128 & 1025 & 250047 & 256298175 & 17 & 210.21 & 12.37 & 7.47 \\
256 & 2049 & 250047 & 512346303 & 17 & 209.56 & 12.33 & 14.90 \\
512 & 4097 & 250047 & 1024442559 & 17 & 210.14 & 12.36 & 29.89 \\
1024 & 8193 & 250047 & 2048635071 & 18 & 221.77 & 12.32 & 63.08 \\
2048 & 16385 & 250047 & 4097020095 & 18 & 221.12 & 12.28 & 125.80 \\
\hline
\end{tabular}

Table 5.5. Weak scaling results for the $3 \mathrm{D}$ problem. 
Acknowledgement. The authors would like to thank their advisor Rob Stevenson for the many fruitful discussions.

Funding. Both authors were supported by Netherlands Organization for Scientific Research (NWO) under contract no. 613.001.652. Computations were performed at the national supercomputer Cartesius under SURF code EINF-459.

\section{References}

And13. Roman Andreev. Stability of sparse space-time finite element discretizations of linear parabolic evolution equations. IMA Journal of Numerical Analysis, 33(1):242-260, 12013.

And16. Roman Andreev. Wavelet-In-Time Multigrid-In-Space Preconditioning of Parabolic Evolution Equations. SIAM Journal on Scientific Computing, 38(1):A216-A242, 12016.

BJ89. Ivo Babuška and Tadeusz Janik. The h-p version of the finite element method for parabolic equations. Part I. The p-version in time. Numerical Methods for Partial Differential Equations, 5(4):363-399, 1989.

BJ90. Ivo Babuška and Tadeusz Janik. The h-p version of the finite element method for parabolic equations. II. The h-p version in time. Numerical Methods for Partial Differential Equations, 6(4):343-369, 1990.

Bra81. Achi Brandt. Multigrid solvers on parallel computers. In Elliptic Problem Solvers, pages 39-83. Elsevier, 11981.

Dij09. TJ Dijkema. Adaptive tensor product wavelet methods for the solution of PDEs. PhD thesis, Utrecht University, 2009.

DPS05. Lisandro Dalcín, Rodrigo Paz, and Mario Storti. MPI for Python. Journal of Parallel and Distributed Computing, 65(9):1108-1115, 92005.

DS18. Denis Devaud and Christoph Schwab. Space-time hp-approximation of parabolic equations. Calcolo, 55(3):35, 92018.

FFK $^{+}$14. R. D. Falgout, S. Friedhoff, Tz. V. Kolev, S. P. MacLachlan, and J. B. Schroder. Parallel Time Integration with Multigrid. SIAM Journal on Scientific Computing, 36(6):C635-C661, 12014.

FK19. Thomas Führer and Michael Karkulik. Space-time least-squares finite elements for parabolic equations. 112019.

Gan15. Martin J. Gander. 50 Years of Time Parallel Time Integration. In Multiple Shooting and Time Domain Decomposition Methods, chapter 3, pages 69113. Springer, Cham, 2015.

GN16. Martin J. Gander and Martin Neumüller. Analysis of a New Space-Time Parallel Multigrid Algorithm for Parabolic Problems. SIAM Journal on Scientific Computing, 38(4):A2173-A2208, 12016.

Hac85. Wolfgang Hackbusch. Multi-Grid Methods and Applications, volume 4 of Springer Series in Computational Mathematics. Springer Berlin Heidelberg, Berlin, Heidelberg, 1985.

HVW95. G. Horton, S. Vandewalle, and P. Worley. An Algorithm with Polylog Parallel Complexity for Solving Parabolic Partial Differential Equations. SIAM Journal on Scientific Computing, 16(3):531-541, 51995.

LMT01. Jacques-Louis Lions, Yvon Maday, and Gabriel Turinici. Résolution d'EDP par un schéma en temps «pararéel ». Comptes Rendus de l'Académie des Sciences - Series I - Mathematics, 332(7):661-668, 42001. 
$\mathrm{MFL}^{+}$91. Oliver A. McBryan, Paul O. Frederickson, Johannes Lindenand, Anton Schüller, Karl Solchenbach, Klaus Stüben, Clemens-August Thole, and Ulrich Trottenberg. Multigrid methods on parallel computers-A survey of recent developments. IMPACT of Computing in Science and Engineering, 3(1):1-75, 31991.

Nie64. J. Nievergelt. Parallel methods for integrating ordinary differential equations. Communications of the ACM, 7(12):731-733, 121964.

NS19. Martin Neumüller and Iain Smears. Time-parallel iterative solvers for parabolic evolution equations. SIAM Journal on Scientific Computing, 41(1):C28-C51, 12019.

OR00. Maxim A. Olshanskii and Arnold Reusken. On the Convergence of a Multigrid Method for Linear Reaction-Diffusion Problems. Computing, 65(3):193202, 122000 .

RS19. Nikolaos Rekatsinas and Rob Stevenson. An optimal adaptive tensor product wavelet solver of a space-time FOSLS formulation of parabolic evolution problems. Advances in Computational Mathematics, 45(2):1031-1066, 42019 .

Sch14. Joachim Schöberl. C ++11 Implementation of Finite Elements in NGSolve. Technical report, Institute for Analysis and Scientific Computing, Vienna University of Technology, 2014.

SS09. Christoph Schwab and Rob Stevenson. Space-time adaptive wavelet methods for parabolic evolution problems. Mathematics of Computation, 78(267):1293-1318, 92009.

Ste98. Rob Stevenson. Stable three-point wavelet bases on general meshes. $\mathrm{Nu}$ merische Mathematik, 80(1):131-158, 71998.

Ste03. Rob Stevenson. Locally Supported, Piecewise Polynomial Biorthogonal Wavelets on Nonuniform Meshes. Constructive Approximation, 19(4):477508, 82003

SvVW21. Rob Stevenson, Raymond van Venetië, and Jan Westerdiep. A wavelet-intime, finite element-in-space adaptive method for parabolic evolution equations. 12021.

SW20. Rob Stevenson and Jan Westerdiep. Stability of Galerkin discretizations of a mixed space-time variational formulation of parabolic evolution equations. IMA Journal of Numerical Analysis, 22020.

SY18. Olaf Steinbach and Huidong Yang. Comparison of algebraic multigrid methods for an adaptive space-time finite-element discretization of the heat equation in 3D and 4D. Numerical Linear Algebra with Applications, 25(3):e2143, 52018.

SZ20. Olaf Steinbach and Marco Zank. Coercive space-time finite element methods for initial boundary value problems. ETNA - Electronic Transactions on Numerical Analysis, 52:154-194, 2020.

Vir20. Pauli Virtanen. SciPy 1.0: fundamental algorithms for scientific computing in Python. Nature Methods, 17(3):261-272, 32020.

vVW20a. Raymond van Venetië and Jan Westerdiep. Efficient space-time adaptivity for linear parabolic evolution equations. 2020.

vVW20b. Raymond van Venetië and Jan Westerdiep. Implementation of: A parallel algorithm for solving linear parabolic evolution equations, 12020.

Wor91. Patrick H. Worley. Limits on Parallelism in the Numerical Solution of Linear Partial Differential Equations. SIAM Journal on Scientific and Statistical Computing, 12(1):1-35, 11991. 